\title{
Frequency noise in frequency swept fiber laser
}

\author{
Pedersen, Anders Tegtmeier; Rottwitt, Karsten
}

Published in:

Optics Letters

Link to article, DOI:

10.1364/OL.38.001089

Publication date:

2013

Document Version

Publisher's PDF, also known as Version of record

Link back to DTU Orbit

Citation (APA):

Pedersen, A. T., \& Rottwitt, K. (2013). Frequency noise in frequency swept fiber laser. Optics Letters, 38(7), 1089-1091. https://doi.org/10.1364/OL.38.001089

\section{General rights}

Copyright and moral rights for the publications made accessible in the public portal are retained by the authors and/or other copyright owners and it is a condition of accessing publications that users recognise and abide by the legal requirements associated with these rights.

- Users may download and print one copy of any publication from the public portal for the purpose of private study or research.

- You may not further distribute the material or use it for any profit-making activity or commercial gain

- You may freely distribute the URL identifying the publication in the public portal

If you believe that this document breaches copyright please contact us providing details, and we will remove access to the work immediately and investigate your claim 


\title{
Frequency noise in frequency swept fiber laser
}

\author{
Anders Tegtmeier Pedersen* and Karsten Rottwitt \\ Department of Photonics Engineering, Technical University of Denmark, Ørsteds Plads 343, Lyngby 2800 Kgs., Denmark \\ ${ }^{*}$ Corresponding author: antp@fotonik.dtu.dk
}

Received January 2, 2013; revised February 21, 2013; accepted February 21, 2013; posted February 21, 2013 (Doc. ID 182143); published March 22, 2013

\begin{abstract}
This Letter presents a measurement of the spectral content of frequency shifted pulses generated by a lightwave synthesized frequency sweeper. We found that each pulse is shifted in frequency with very high accuracy. We also discovered that noise originating from light leaking through the acousto- optical modulators and forward propagating Brillouin scattering appear in the spectrum. (c) 2013 Optical Society of America

OCIS codes: $\quad 060.2630,060.3510$.
\end{abstract}

A lightwave synthesized frequency sweeper (LSFS) is a fiber optical configuration that uses a frequency shifter in a recirculation loop to obtain a linear frequency sweep of the laser pulses emitted. The resulting output is a train of frequency-shifted pulses. The LSFS was first described in 1990 [1] and has since been described in a number of applications, including WDM systems [2], medical imaging [3], dispersion measurements [4], and in remote sensing [ [ $[$ ]. Until now the main emphasis has been on maximizing the frequency sweep range and optimizing the output power stability, which minimizes the noise. Sweeping ranges exceeding $1 \mathrm{THz}$ and stable operation over several hours have been reported $[\underline{6}, \underline{7}]$.

Most LSFSs reported in the literature rely on acoustooptic modulators (AOMs) to shift the frequency of the light because AOMs have high extinction ratios, often in excess of $50 \mathrm{~dB}$, and provide high-precision frequency shifts determined by the RF frequency feeding the AOM. Using a stable laser source and RF generators, a spectrally stable optical output is obtained. The frequency shift, however, is often in the order of tens or maybe hundreds of megahertz, which is small compared to the approximately $193 \mathrm{THz}$ carrier frequency of a laser emitting around $1.55 \mu \mathrm{m}$. Hence, a considerable number of frequency shifts is necessary to obtain a sweep of even $1 \mathrm{~nm}$.

Theoretically, the LSFS was successfully modeled in [8] by assuming that the total power in the ring configuration is always constant. This model has been further developed in later publications to describe various changes to the basic configuration such as different signal wavelengths or sweeping bandpass filters (BPFs) $[\underline{3}, \underline{7}, \underline{9}]$. The advantage of this model is its simplicity; it makes cumbersome rate equations unnecessary and instead allows the use of simple propagation equations to describe the development of signal and noise, coupled through the gain of the amplifier in the loop. However, there also is a drawback: since any time dependencies are disregarded, information on the shape of the individual pulses is lost. Using time-dependent rate equations to describe the LSFS was used in [10] and excellent agreement between simulations and measurements, even on the individual pulses, was found. This agreement, however, came at the expense of longer computation time.

Here we measure the LSFS output in the frequency domain to investigate the frequency sweep accuracy as well as frequency-dependent noise contributions.

Figure 1 shows a sketch of the LSFS used. The setup is an all-fiber ring configuration consisting of a $3 \mathrm{~dB}$ coupler
(1) in the figure), a commercial erbium-doped fiber amplifier (EDFA), an BPF with a $3 \mathrm{~dB}$ width of $0.84 \mathrm{~nm}$, an $\mathrm{AOM}$ (2), an $11 \mathrm{~km}$ delay line, and a polarization controller (PC). The ring is initially seeded by a pulse produced by an $\mathrm{AOM}$ (3) outside the ring from the continuous output of a narrow linewidth, $1548 \mathrm{~nm}$ fiber laser. The pulse length is matched to be approximately 500 ns shorter than the ring round-trip time so that the entire pulse fits within the ring without overlapping with itself. The $3 \mathrm{~dB}$ coupler directs half of the power of the seed pulse to the output and the other half into the ring. In the ring, the EDFA is used to compensate for the transmission and coupling loss, the BPF is used to eliminate broadband amplified spontaneous emission (ASE) from the EDFA, and the AOM shifts the carrier frequency of the light by $40 \mathrm{MHz}$.

The delay line, which is just a length of single-mode optical fiber, is used to adjust the length of the ring to match the desired pulse length and is chosen freely, though in the lower limit the length is constrained by the switching time of the seed AOM. After a full revolution, the power is again divided by the $3 \mathrm{~dB}$ coupler and half is directed to the output and half is redirected into the ring where it is re-amplified and the frequency is shifted again. If the pulse length is properly matched with the ring round-trip time the resulting output power is nearly constant in time whereas the carrier frequency is shifted in steps corresponding to the AOM frequency shift at a rate corresponding to the round-trip time.

The operation of the loop with respect to the number of pulses in a pulse train is limited by the BPF in combination with the build-up of ASE. The EDFA has build-in isolators ensuring unidirectional operation for both

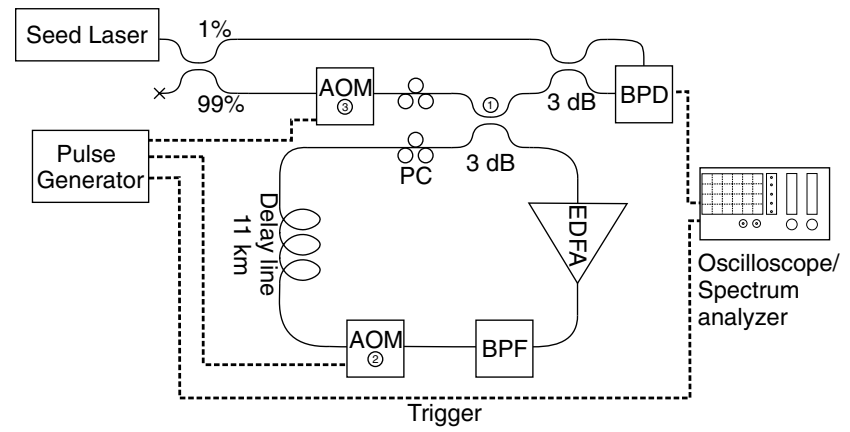

Fig. 1. Setup used to generate the FSPT and measure the spectrum of the individual pulses. 
signal and ASE. However, in the forward direction, ASE builds up over time and deteriorates the signal-to-noise ratio. Since ASE is relatively broad banded the buildup is impeded by the use of a BPF around the signal frequency; the narrower the filter the more ASE it eliminates, but fewer frequency shifts are possible before the signal itself is attenuated. Once this happens it is necessary to restart the LSFS laser ring by closing the AOM inside the ring and thus terminating all light in the ring. This action generates a new seed pulse and the entire process is repeated.

To measure the spectrum of the individual pulses in the frequency-stepped pulse train (FSPT), we use a heterodyne measurement with a balanced photodetector (BPD) and an electrical spectrum analyzer (ESA) (Fig. 1). One percent of the seed laser power is bypassed the ring and subsequently joined with the resulting pulses at the ring output. This local oscillator signal is necessary for the heterodyne measurement. A time gate is used to measure one and only one pulse at a time. Every time a new pulse train is initiated a trigger signal goes to the ESA, which then measures the pulse for a given length of time (e.g., $10 \mu \mathrm{s}$ ). By changing the time from when the trigger signal is received to when the measurement is initiated, each individual pulse can be measured.

The LSFS described above was used to generate a pulse train consisting of 15 individual pulses, each $50 \mu \mathrm{s}$ long, for the spectral analysis. Figure 2 shows an example of the spectrum of the 11th pulse in the pulse train measured from 10 to $640 \mathrm{MHz}$ with a resolution bandwidth of $390 \mathrm{kHz}$ and averaged over 200 pulses. The averaging makes stable features that are representative of many pulse trains stand out, while nonstable features might be lost in the averaging.

The main peak is readily seen at $440.0 \mathrm{MHz}$ with a power level of $-3.23 \mathrm{dBm}$, in excellent agreement with 11 frequency shifts of $40 \mathrm{MHz}$, thereby confirming the ability of the AOM to accurately shift the frequency. This peak is very narrow with a $3 \mathrm{~dB}$ width less than the bandwidth of the measurement owing to the narrow linewidth of the seed laser.

Beside the main peak, two distinct features stand out in the spectrum. The first is the narrow peaks seen equidistantly separated on either side of the main peak and marked using $*$. The spacing between these peaks is $40.0 \mathrm{MHz}$, which strongly indicates that they are related to the AOMs (i.e., light that is either non- or double-shifted

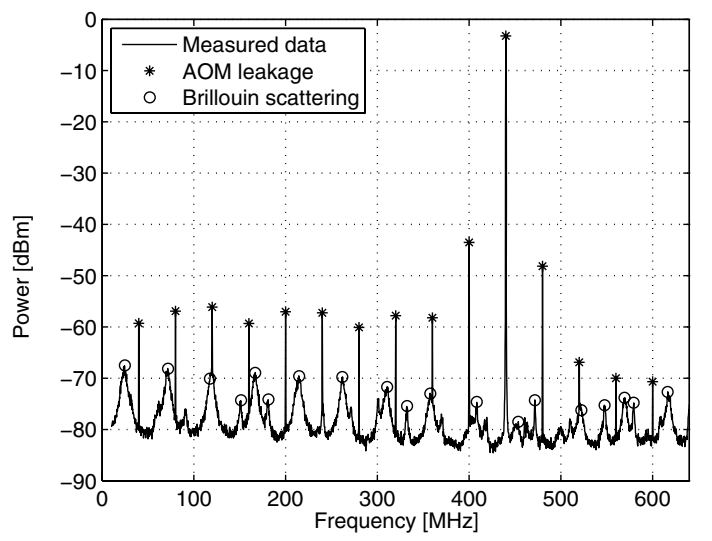

Fig. 2. Measured power versus frequency of the 11th pulse. in frequency during transmission through the modulators). Most prominent of these peaks are those two immediately next to the main peak at $400 \mathrm{MHz}$ and $480 \mathrm{MHz}$ with peak values of $-43.52 \mathrm{dBm}$ and $-48.14 \mathrm{dBm}$, respectively. Below $400 \mathrm{MHz}$, the height of these peaks lies between $-60 \mathrm{dBm}$ and $-56 \mathrm{dBm}$, but with small variations that seem to repeat every $120 \mathrm{MHz}$. For frequencies higher than the main peak, the height of the peaks gradually decreases, but they are nevertheless visible in the entire measured spectrum.

The second distinct feature is a series of low, but much wider, peaks that are marked here with open circle $(\circ)$. The first is centered around $24.4 \mathrm{MHz}$ and has a $3 \mathrm{~dB}$ width of approximately $6 \mathrm{MHz}$. The following peaks are of similar width and separated by about $48 \mathrm{MHz}$, but their height tends to decrease slightly at frequencies closer to the main peak. These peaks are due to scattering of light off radial acoustic modes in the fiber and are therefore due to an interaction between the propagating light and a shear mode of the Brillouin scattering. The acoustic modes arise spontaneously due to the finite temperature of the fiber and their existence is supported by the cylindrical geometry of the fiber [11]. Contrary to the perhaps better known stimulated Brillouin scattering, which travels in the backward direction, shear modes of the Brillouin scattering result in forward propagating waves that are detected in the experimental setup used here. When propagating in the LSFS, the Brillouin scattered light also is frequency shifted by the AOM and, for every revolution, the shift of the main signal frequency leads to generation of new peaks. This action makes the spectrum of the Brillouin scattering complex and varying from pulse to pulse. However, in the case of light propagating in a single optical fiber, the frequency of each acoustic mode and therefore also the frequency shift of the scattered light may be calculated from

$$
\Omega_{m}=\frac{V_{s} y_{m}}{2 \pi a}
$$

where $V_{s}$ is the velocity of the transverse acoustic waves in the fiber, $a$ is the total fiber radius (core plus cladding radius), and $y_{m}$ is the $m$ th zero of the following determinant [11]:

$$
\left|\begin{array}{cc}
\left(3-y^{2} / 2\right) J_{2}\left(\frac{V_{s}}{V_{d}} y\right) & \left(6-y^{2} / 2\right) J_{2}(y)-3 y J_{3}(y) \\
J_{2}\left(\frac{V_{s}}{V_{d}} y\right)-\frac{V_{s}}{V_{d}} y J_{3}\left(\frac{V_{s}}{V_{d}} y\right) & \left(2-y^{2} / 2\right) J_{2}(y)+y J_{3}(y)
\end{array}\right|
$$

Here $J_{2}$ and $J_{3}$ are Bessel functions of the first kind, order 2 and 3 , respectively, and $V_{d}$ is the velocity of the longitudinal acoustic waves in the fiber. The efficiency of the scattering is proportional to the fiber length, and using a shorter delay line could thus reduce the influence of the Brillouin scattering. It is further noted that the experiment showed a strong polarization dependency of the scattering efficiency.

To confirm that Brillouin scattering is the cause of the detected peaks the $11 \mathrm{~km}$ delay line was removed from the setup and the spectrum of light transmitted through 


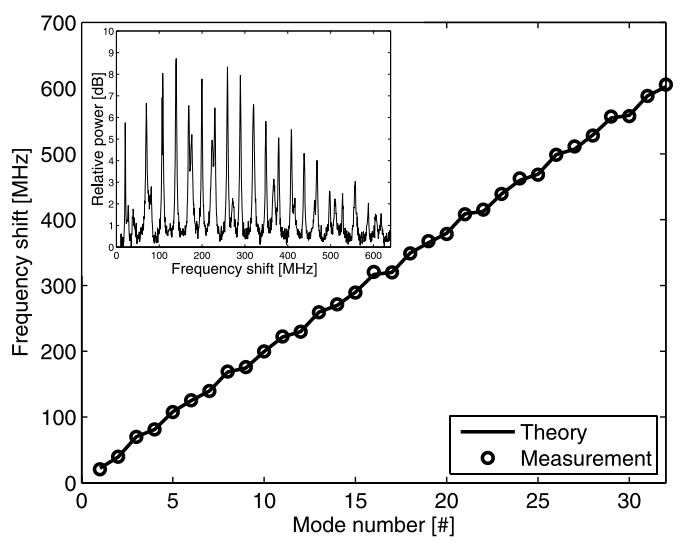

Fig. 3. Comparison of calculated and measured frequency shift due to radial acoustic modes in the fiber. Inset: measured spectrum of forward-propagating Brillouin scattering in the $11 \mathrm{~km}$ long delay line.

the fiber recorded. The inset in Fig. 3 shows the resulting spectrum after subtraction of background noise and several distinct peaks are easily seen. The main figure in Fig. 3 shows the frequency shift of the first 32 modes calculated using Eq. (1) (solid line) together with the measured frequency shifts (open circle) found from the inset figure. In the calculation, values of $3764 \mathrm{~m} / \mathrm{s}$, $5968 \mathrm{~m} / \mathrm{s}$, and $63.1 \mu \mathrm{m}$ were used for $V_{s}, V_{d}$, and $a$, respectively, and the latter was used as a fitting parameter, resulting in a value in very good agreement with the expected value of $62.5 \mu \mathrm{m}$ [12].

As seen in Fig. 3, excellent agreement between the calculated and measured frequency shifts is found throughout the measured spectrum and also the spacing between the peaks agrees very well with the spacing found in the pulse spectra. This confirms the origin of the noise peaks. Besides using a shorter fiber, one way to reduce the Brillouin scattering could be to use a different fiber type, such as a photonic crystal fiber [13].

A LSFS has been build to investigate the spectral content of the emitted pulses and especially the accuracy of the frequency shifts. The generated stable pulse trains consisted of 15 pulses, each being $50 \mu$ s wide. The spectral content was measured in a heterodyne setup with a balanced photo detector and a time-gated ESA.
The measurements showed that the shift in frequency from pulse to pulse determined by the AOM, as expected, was extremely accurate and better than the resolution bandwidth used in the measurement. However, the measurements also revealed different noise contributions in the spectra of the pulses. First, the AOM leaks nonshifted as well as double-shifted light leading to narrow spikes at integer multiples of the AOM RF frequency. Second, the optical fiber itself gives rise to noise in the form of frequency-shifted light originating from forward-going Brillouin scattering. These noise contributions are small, but can nevertheless have a severe effect where quantum noise limited detection is necessary such as, for example, in wind-sensing Doppler lidar.

\section{References}

1. P. Coppin and T. Hodgkinson, Electron. Lett. 26, 28 (1990).

2. K. Aida and K. Nakagawa, J. Lightwave Technol. 14, 1153 (1996).

3. F. D. Nielsen, L. Thrane, P. E. Andersen, J. F. Black, and A. Bjarklev, Opt. Express 13, 4096 (2005).

4. H. Takesue and T. Horiguchi, J. Lightwave Technol. 20, 625 (2002).

5. P. Lindelöw and J. J. Mohr, in International Geoscience and Remote Sensing Symposium (IGARSS) (IEEE, 2007), p. 2787.

6. H. Takesue, F. Yamamoto, K. Shimizu, and T. Horiguchi, Electron. Lett. 34, 1507 (1998).

7. H. Takesue and T. Horiguchi, J. Lightwave Technol. 22, 755 (2004).

8. K. Shimizu, T. Horiguchi, and Y. Koyamada, Appl. Opt. 33, 3209 (1994).

9. K. Takano, K. Nakagawa, and H. Ito, Opt. Express 14, 10313 (2006).

10. A. T. Pedersen, A. S. Olesen, and K. Rottwitt, J. Opt. Soc. Am. B 28, 1493 (2011).

11. R. M. Shelby, M. D. Levenson, and P. W. Bayer, Phys. Rev. B 31, 5244 (1985).

12. W. M. Haynes (ed.), CRC Handbook of Chemistry and Physics: A Ready-Reference Book of Chemical and Physical Data, 93rd ed. (CRC, 2012).

13. D. Elser, U. L. Andersen, A. Korn, O. Glockl, S. Lorenz, Ch. Marquardt, and G. Leuchs, Phys. Rev. Lett. 97, 133901 (2006). 